BMJ Nutrition, Prevention \& Health

\section{Genetic variants for personalised management of very low carbohydrate ketogenic diets}

To cite: Aronica L, Volek J, Poff A, et al. Genetic variants for personalised management of very low carbohydrate ketogenic diets. BMJ Nutrition, Prevention \& Health 2020;3:e000167. doi:10.1136/ bmjnph-2020-000167

- Additional material is published online only. To view, please visit the journal online (http://dx.doi.org/10.1136/ bmjnph-2020-000167).

${ }^{1}$ Nutrition Science, Metagenics Inc, Gig Harbor, Washington, USA ${ }^{2}$ Medicine, Stanford University, Stanford, California, USA

${ }^{3}$ Human Sciences, The Ohio State University, Columbus, Ohio, USA

${ }^{4}$ Medicine Molecular Pharmacology \& Physiology, University of South Florida, Tampa, Florida, USA

Correspondence to Dr Lucia Aronica, Medicine, Stanford University, Stanford, CA 94305, USA;

lucia.aronica@metagenics.com

Received 7 September 2020 Revised 4 November 2020 Accepted 15 November 2020 Published Online First 12 December 2020

Check for updates

(c) Author(s) (or their employer(s)) 2020. Re-use permitted under CC BY-NC. No commercial re-use. See rights and permissions. Published by BMJ.

\section{ABSTRACT}

The ketogenic diet (KD) is a low-carbohydrate, highfat, adequate-protein diet proven to be effective for the reversal of obesity, metabolic syndrome and type 2 diabetes, and holding therapeutic potential for the prevention and treatment of other chronic diseases. Genetic and dynamic markers of KD response may help to identify individuals most likely to benefit from KD and point to individuals at higher risk for adverse health outcomes. Here, we provide a clinician-friendly review of state-of-theart research on biomarkers of $\mathrm{KD}$ response for a variety of outcomes including weight loss, body composition and cognitive performance drawing data from both intervention trials and case reports of rare inborn errors of metabolism. We also present a selection of the most promising candidate genes to evaluate in future studies and discuss key aspects of study design and variant interpretation that may help accelerate the implementation of these biomarkers in clinical practice.

\section{INTRODUCTION}

As the field of precision medicine is gaining traction, nutrition research is experiencing a 'gold rush' for biomarkers that may enable the selection of personalised dietary interventions to maximise an individual's likelihood of successful response. These biomarkers include both genetic factors and dynamic biomarkers that respond to lifestyle factors such as physiological markers, ${ }^{1}$ epigenetics and transcriptomics, ${ }^{2}$ metabolomics ${ }^{3}$ and the microbiome. ${ }^{4}$ The use of biomarkers of dietary response would be of particular clinical relevance for the selection and individualised risk-benefit analysis of therapeutic diets, such as the ketogenic diet (KD), which provide alternative or complementary therapies to standard-of-care treatments.

$\mathrm{KD}$ is a low-carbohydrate, high-fat, adequateprotein diet that was historically used to treat epilepsy and diabetes a century ago prior to the discovery of insulin and antiseizure medications. ${ }^{56}$ The latest scientific evidence showing that ketones are both preferred fuels and potent signalling molecules has dramatically increased the number of investigations into the basic science of ketones and their applications across broad disciplines. Ketones demonstrate pleiotropic actions affecting gene expression and pathways regulating inflammation, oxidative stress, immune function, membrane health, cell signalling and antioxidant status ${ }^{7}$ that manifest in reversal of metabolic disease and extended healthspan. The most remarkable therapeutic evidence of KDs is the rapid and sustained reversal of obesity, metabolic syndrome (MetS) and type 2 diabetes in a plethora of recent published research. Preclinical and other evidence point to KDs as a promising therapeutic intervention in other chronic diseases including Alzheimer's disease (AD) and certain type of cancers. ${ }^{8} \mathrm{KD}$ induces a metabolic switch from using glucose to using fat as primary fuel for the body. There are different variations of KD with the most commonly used protocols recommending 30-50 g or less of dietary carbohydrate or 25-30 g of 'net carbs' (calculated by total carbohydrate minus fibre) per day. ${ }^{9} 10$ Beta-hydroxybutyrate (BHB) and acetoacetate are the two main ketone bodies in blood that are produced from partial oxidation of fatty acids. Both ketone bodies and fatty acids are important sources of energy for the brain, heart, muscle, kidney and other tissues when glucose availability is limited such as during periods of carbohydrate restriction and fasting or when energy demands are increased, such as during illness or vigorous exercise.

A personalised lifestyle approach to KD would enable to maximise both therapeutic effectiveness and long-term safety for patients. Although KD has proven to be effective and safe for most people in trials up to 2 years, ${ }^{11}$ the degree of therapeutic response in terms of weight loss, metabolic changes and neurological effects varies across individuals. ${ }^{8}{ }^{12}$ In addition to physiological factors such as sex and age, this variability likely reflects the interaction of genetic and lifestyle-related 
factors including diet, insulin resistance (IR) and activity level. As $\mathrm{KD}$ and personalised nutrition are becoming more and more popular, genetic variants for the prediction of KD response are increasingly discussed in media articles, blog posts and direct to consumer genetic reports. However, most of these variants have not been evaluated in intervention trials of $\mathrm{KD}$, which can mislead and confuse consumers and their health practitioners.

The main aim of this review is to provide clinicians and patients with a snapshot of clinically tested common single-nucleotide polymorphisms (SNPs) for the prediction of $\mathrm{KD}$ response, ranked by their strength of scientific evidence. These SNPs have been identified in intervention trials testing the effects of $\mathrm{KD}$ on various outcomes including weight loss, body composition and cognitive performance. In addition, we also provide a list of rare mutations with strong effects on KD response, either as a therapeutic indication or contraindication, and present a selection of the most promising candidate genes and approaches to evaluate in future studies. Finally, we discuss the importance of establishing common standards of study design and variant interpretation and delineate the steps we need to take towards the implementation of practice-based guidelines.

\section{COMMON SNPS ASSOCIATED WITH METABOLIC OR NEUROLOGICAL OUTCOMES IN INTERVENTION STUDIES OF KD OR KETOGENIC AGENTS}

Genetic variation may influence KD response by affecting the body's ability to process and use carbohydrates or fats. While rare mutations (frequency $<1 \%$ ) can produce strong effects on phenotype, common (SNPs, minor allele frequency $\geq 1 \%$ ) result in more subtle effects that depend on the interaction with other genetic variants and environmental factors. Genome-wide association studies (GWAS) have identified many SNPs associated with interindividual differences in the response to high fat food intake. However, most of these GWAS were observational studies based on populations that consumed diets high in both fats and carbohydrates, often referred as to obesogenic high-fat diets (oHFD). ${ }^{13}$ oHFD-associated SNPs, such as those in the PPARA gene, ${ }^{14}$ are commonly but misleadingly listed as a contraindication to $\mathrm{KD}$ in many genetic interpretation tools available on the internet. Since the metabolic, hormonal and neurological effects of oHFDs are diametrically opposite to those of $\mathrm{KD}$, we do not have any evidence for the use of oHFD-associated SNPs for the prediction of $\mathrm{KD}$ response.

Only a few intervention studies have tested the effects of oHFD-associated SNPs on weight loss or body composition in the context of a carbohydrate restriction sufficient to induce ketosis. In table 1, we provide a list of these SNPs as well as other SNPs that have been associated with interindividual differences in the neurological response to $\mathrm{KD}$ or ketogenic agents in other intervention studies. A strength of evidence score is provided for each of these
SNPs based on recent guidelines for the interpretation of nutrigenetic variants. ${ }^{15}$

\section{SNPs associated with weight loss and body composition outcomes}

A study in 86 adults (53 overweight men and 33 normoweight/overweight women) found that SNPs in genes encoding for the metabolic enzymes gastric lipase (LIPF, rs814628-G), hepatic glycogen synthase (GYS2, rs2306179-C), cholesteryl ester transfer protein (rs5883-T) and galanin (rs694066-G) were significantly associated with a greater weight loss in response to $\mathrm{KD}$ (8\%-13\% CHO, 60\%-63\% fat and 28\%-30\% protein) over a period of $4-12$ weeks. ${ }^{16}$

Another study compared the effects of $\mathrm{KD}(\mathrm{n}=93$, CHO $\sim 12 \%$ ) vs a low fat diet (LF, $\mathrm{n}=70$, fat $\sim 25 \%$ ) on weight loss and body composition, and the association of these effects with genetic variants. The minor $\mathrm{G}$ allele of the rs5950584 SNP in the promoter region of the angiotensin II receptor type 2 (AGTR2) gene was associated with a greater reduction in body fat percentage in response to KD (CHO 12\%). ${ }^{17}$ The AGTR2 gene has been implicated in the accumulation of fat and development of muscle cell IR induced by high fat, hypercaloric feeding in mice. ${ }^{18}$ The AGTR2 gene is X-linked, thus men can have only one of either the $\mathrm{T}$ (major) or the $\mathrm{G}$ (minor) allele, while women may have zero, one or two copies of either allele. This means that the effect of this gene variant on fat loss with $\mathrm{KD}$ may be more prevalent and/ or stronger in men than in women. The major $\mathrm{G}$ allele in the rs322695 SNP of the RARB gene was associated with greater reductions in body fat percentage within both the $\mathrm{KD}$ and LF groups. The RARB gene encodes the retinoic acid receptor beta, a thyroid-steroid hormone receptor which regulates energy production in the liver through modulation of gene expression. This observation is noteworthy in light of the putative role of the retinoic acid system in IR. ${ }^{19}$ Other genetic variants were associated with a greater reduction in body fat percentage within the LF group only. These include the major $\mathrm{G}$ allele of the rs12691940 SNP in the HNMT gene, which has been implicated in appetite regulation in mice, ${ }^{20}$ and the major $\mathrm{G}$ allele of the rs2838549 SNP in the PFKL gene, which produces a key regulatory enzyme for glycolysis known as hepatic phosphofructokinase.

\section{SNPs associated with neurological outcomes}

Using a discovery approach, a recent intervention study in epileptic children found that a SNP within the chromodomain Y 1 ligand, CDY1L, may affect the seizure $\mathrm{KD}$ response. ${ }^{21} \mathrm{CDY} 1 \mathrm{~L}$ is a chromodomain protein and histone acetyltransferase with important roles in the epigenetic regulation of gene expression in the brain. In this study, 232 epileptic children (age 3-9; male, $\mathrm{n}=131$; female, $\mathrm{n}=121 ; 82 \%$ Caucasians) received $\mathrm{KD}$ for 3 months (classical KD, $\mathrm{n}=165$; medium chain triglyceride (MCT) KD, n=48; modified KD, $\mathrm{n}=38$ ). Children carrying at least one copy of the CDYL1 rs12204701-A allele, who 


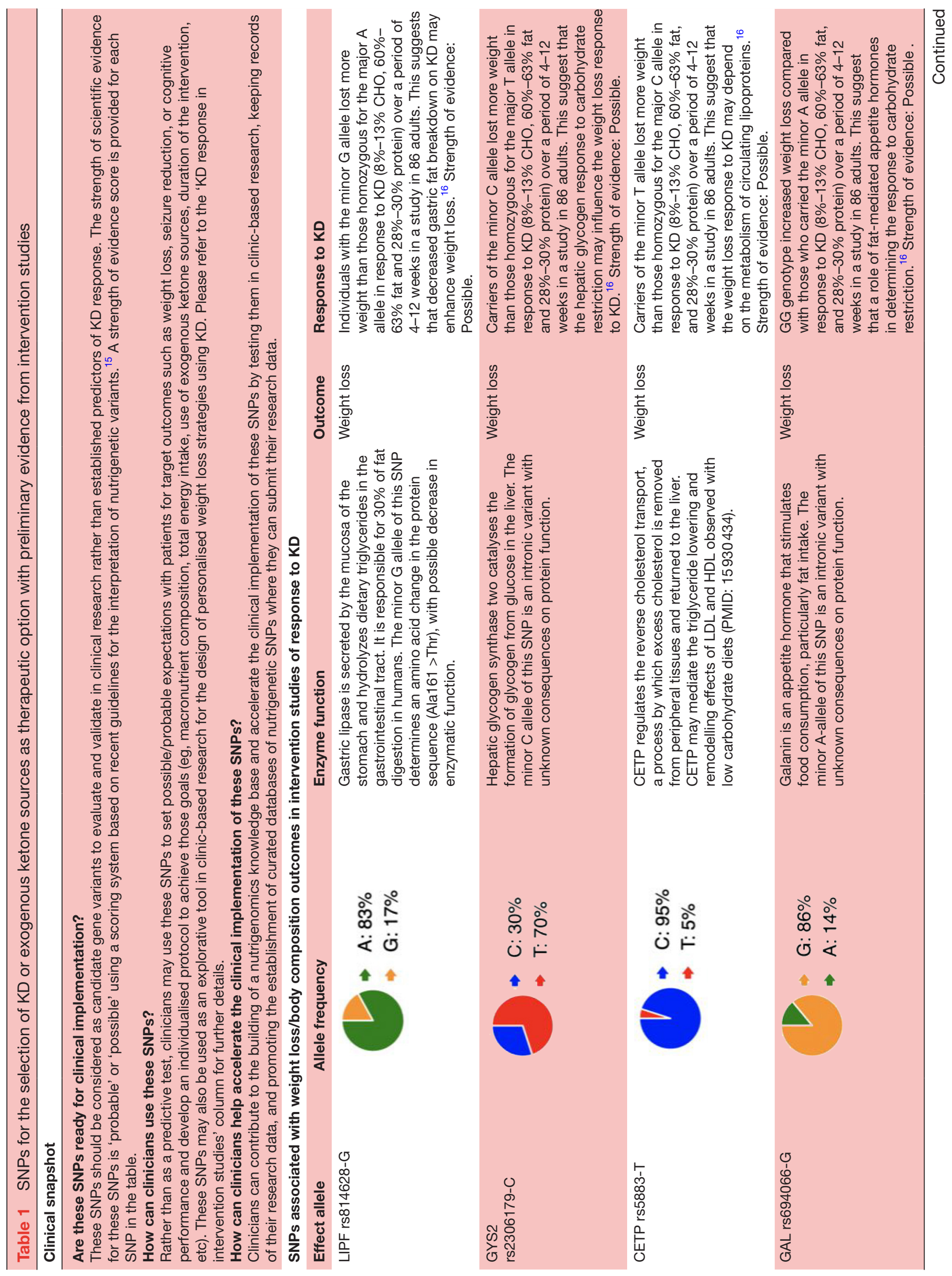




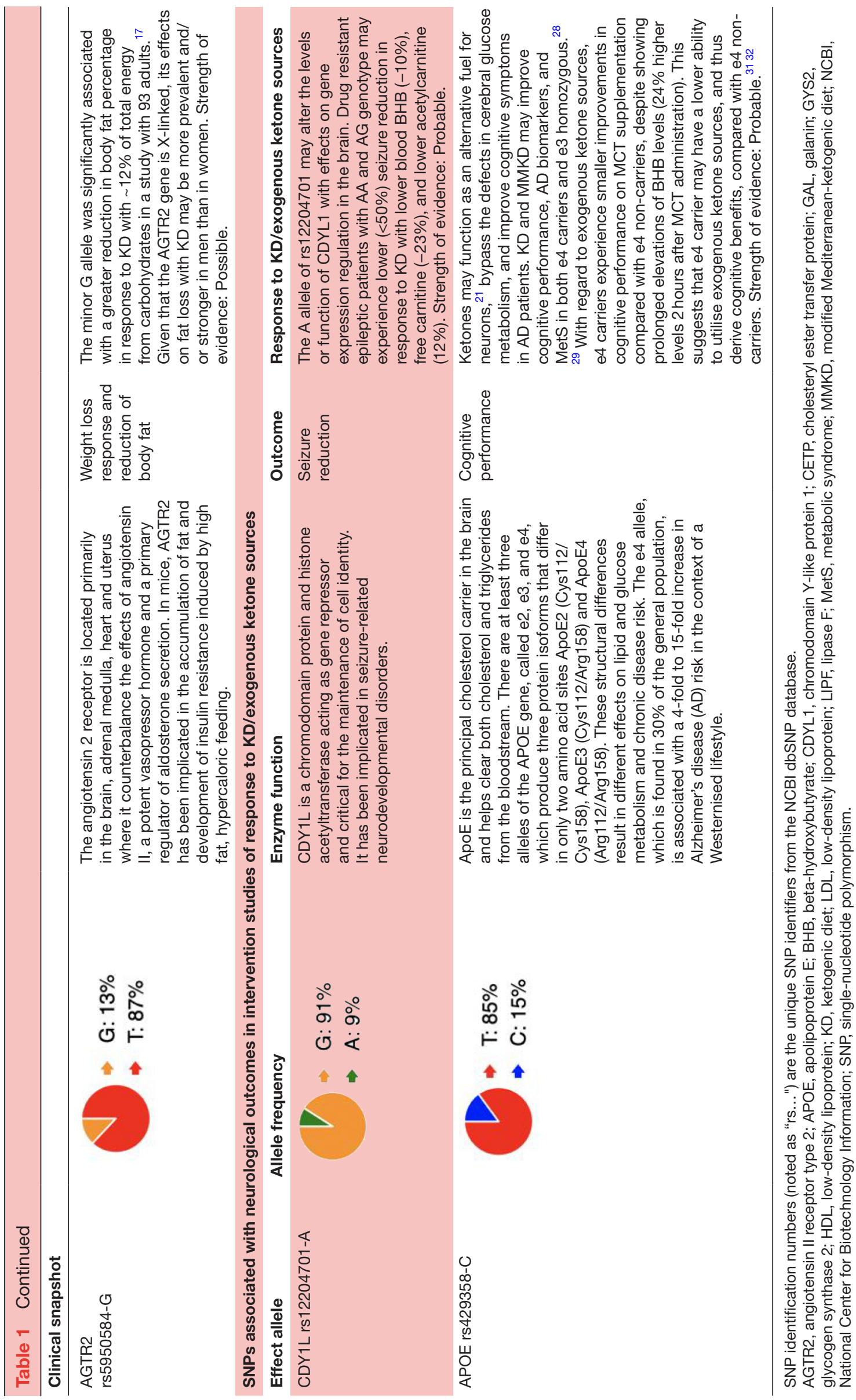


represent $17 \%$ of the general population and almost $50 \%$ of Africans, experienced lower $(\leq 50 \%)$ seizure reduction when following KD compared with non-carriers. The underlying mechanisms of this gene-diet interaction are unknown but might involve differences in KD-mediated gene expression changes in the brain.

Other neurological studies have used a candidate-gene approach to investigate the effects of genetic variation of the apolipoprotein E gene (APOE) on cognitive performance in response to $\mathrm{KD}$ or ketogenic agents in $\mathrm{AD}$ patients. ApoE is the principal cholesterol carrier in the brain and helps clear both cholesterol and triglycerides from the bloodstream. About $30 \%$ of people have at least one copy of the e 4 allele, which has been associated with a 4-fold to 15-fold increase in $\mathrm{AD}$ risk compared with individuals with the more common e3 allele, while the e2 allele is related to decreased risk. ${ }^{22}$ However, lifestyle plays a major role in modifying e4-associated AD risk as illustrated by the observation that, although the e4 allele is most frequently seen in African populations, the incidence of $\mathrm{AD}$ in elderly Nigerians is extremely low. ${ }^{23} \mathrm{E} 4$ carriers are more likely to develop $\mathrm{AD}$ if they have high IR, ${ }^{24}{ }^{25}$ which has been proposed to interact with the e4 variant to produce impaired glucose metabolism, insulin sensitivity and lipid transport in the brain leading to accumulation of amyloid beta and ultimately cognitive failure. ${ }^{26}$ The underlying mechanisms of this interaction are not fully understood and may reflect biochemical differences between different ApoE isoforms such as susceptibility to glycosylation, effects on brain glucose metabolism through regulation of cytosolic hexokinase, and delivery of lipids to the brain. ${ }^{27}$ The KD could alleviate the effects of these alterations and improve cognitive symptoms in patients with $\mathrm{AD}$ by reducing insulin signalling in peripheral tissues and the brain and providing ketones as an alternative energy source.

Preliminary evidence indicates that APOE genotype may affect cognitive performance in response to elevations in blood ketones depending on the modality of how ketosis is achieved (ie, through KD or ketogenic supplements), which can affect the kinetics of cellular uptake of ketone bodies and their blood levels. In a randomised, double-blind, cross-over trial comparing a modified Mediterranean-ketogenic diet (MMKD) vs American Heart Association Diet over a period of 6 weeks, MMKD improved $\mathrm{AD}$ biomarkers such as accumulation of brain amyloid in both e4 carriers and e3 homozygous with mild cognitive impairment (MCI) ( $\mathrm{n}=17$; age 58-70 y; 11 subjects with MCI and 6 cognitive normal) with no significant difference based on APOE genotype. ${ }^{28}$ This is in line with a case report of an e4 heterozygous 71-year-old woman with mild $\mathrm{AD}$ and $\mathrm{MetS}$ who experienced significant improvements in both cognitive performance and MetS biomarkers (HOMA-IR, $-75 \%$; triglycerides, $-50 \%$; very low-density lipoprotein (LDL), $-50 \%$; Hemoglobin $\mathrm{A} 1 \mathrm{C}$ (HgA1c), from $5.7 \%$ to $4.9 \%$ ) after a 10-week protocol of $\mathrm{KD}$, time-restricted eating and physical/ cognitive exercise. ${ }^{29}$ In contrast, greater carbohydrate intake has been associated with poorer performance in attention in e 4 carriers and poorer performance in verbal memory in e4 non-carriers. ${ }^{30}$

On the other hand, the e 4 variant may have an effect on cognitive performance in response to ketogenic agents in patients with $\mathrm{AD}$. In a placebo-controlled trial in 20 patients with $\mathrm{AD}$ or MCI, the administration of MCT, $40 \mathrm{~mL}$ blended with $152 \mathrm{~mL}$ heavy whipping cream produced improvements in cognitive performance as measured by AD Assessment Scale-Cognitive Subscale (ADAS-cog) only in e3 homozygous but not in e4 carriers. ${ }^{31}$ At the same time, e4 carriers experienced, counterintuitively, more prolonged elevations in ketone levels in response to MCT administration suggesting that their lower cognitive response may reflect lower cellular uptake or utilisation of ketogenic agents compared with e4 non-carriers. These findings were replicated in a larger randomised, doubleblind, placebo-controlled study in $152 \mathrm{AD}$ patients, which tested the cognitive effects of an MCT-based supplement composed of glycerin and caprylic acid (AC1202, $10 \mathrm{gr}$ ) administered over a period of 3 months. While AC1202 induced significant improvement in ADAS-cog in both e4 carriers and non-carriers, these effects were greater and significantly correlated with blood BHB levels only in e4 non-carriers. ${ }^{32}$

\section{SNPs associated with blood lipids and cardiovascular health outcomes}

One of the most clinically relevant and frequently asked question among health practitioners and patients is whether some genetic variant may predispose to cardiovascular disease (CAD) such as high LDL cholesterol (LDL-C) in the context of KD. Although several cholesterol-raising genetic variants have been identified and associated with higher CAD risk in the content of oHFD, there is still no evidence about whether these variants may constitute a contraindication to $\mathrm{KD}$. Box 1 provides a brief discussion of this topic along with some practical tips for clinicians managing patients who experience an increase in LDL-C in response to KD.

\section{INBORN GENETIC CONDITIONS WITH EFFECTS ON KD RESPONSE}

While only a few common SNPs have been shown to affect $\mathrm{KD}$ response in clinical trials, several rare mutations (frequency $\leq 1 \%$ ) can cause inborn errors of metabolism (IEM) and have strong effects on KD response, representing either an indication or contra-indication for its use. Online supplemental tables S1 and S2 provide an overview of these conditions and a selection of their most frequently reported mutations with a focus on their effects on $\mathrm{KD}$ response and levels of ketones and glucose in the blood.

$\mathrm{KD}$ is contraindicated and potentially lethal in individuals affected by IEMs that prevent the body from using ketones or fatty acids as fuel such as inborn defects of ketone metabolism as well as fatty acid oxidation disorders 
Box 1 Cholesterol-raising genetic variants: a contraindication for ketogenic diet (KD)?

Approximately one in four people experience increases in low-density lipoprotein cholesterol (LDL-C) when following KD. These individuals are often referred as to 'high responders'. While the biological mechanisms of this response are still unclear, we know that certain genetic variants may predispose some people to produce more cholesterol or reduce its blood clearance on a high fat diet. Several LDL-C-raising common single-nucleotide polymorphisms (SNPS) and rare mutations have been identified and linked to metabolic and cardiovascular disease in the context a Westernised obesogenic high fat diet (OHFD) high both in fats and refined carbohydrates. Examples of well-characterised common SNPs associated with higher LDL-C and/or adverse metabolic traits in the context of oHFD include lipase C, hepatic type (LIPC) rs1800588 (high LDL/high-density lipoprotein (HDL) ratio), ${ }^{50-52}$ AP0A2 rs5082 (high body mass index), ${ }^{37}$ 53-55 APOA4 rs675 (higher LDL-C and ApoB), ${ }^{56}$ PPAR-alpha rs1800206 (high LDL-C and triglycerides (TG) with high saturated fat intake and low polyunsaturated fat intake), ${ }^{14515758}$ as well as non-coding variants such SORT1 rs12740374 (high LDL-C and very LDL particle number). ${ }^{59}$ Examples of rare LDL-C raising variants are those with genes implicated in familial hypercholesterolemia (FH) and other phenotypically related lipid disorders such as LDLR, APOB, PCSK9, LDLRAP1, ${ }^{60-62}$ LIPA, $^{63}$ ABCG5, ABCG8 ${ }^{64}$ and apolipoprotein $\mathrm{E}^{6566}$

However, there is still no evidence about whether these variants may constitute a contraindication to KD, although some direct-to-consumer genetic reports make these claims. Some studies indicate that the increase in LDL-C observed on KD and other low-carb high-fat diets reflects an increase in LDL-C particle size rather than number, a change that is associated with reduced cardiovascular risk and accompanied by an improvement in other cardiovascular risk factors such as TG and HDL. ${ }^{67-70}$ On the other hand, even large LDL-particles can become atherogenic in presence of genetic factors that reduce LDL clearance such in the case of $\mathrm{FH}$ and other lipid disorders.

Therefore, we still do not have an answer to the question clinicians care most about: What patients would do better on KD and what patients would do worse? In absence of evidence-based guidelines, clinicians who manage patients who are 'hyper-responders' to KD typically follow a pragmatic approach involving the assessment of additional cardiovascular markers (eg, LDL particle number, TG, HDL, coronary calcium score) as well as family history of cardiovascular disease. If these markers are altered, patients may consider stopping KD or starting a cholesterol lowering medication. With patients who express a desire and willingness to give $\mathrm{KD}$ a last chance, a common practice is to have them reduce the amount of red meat ${ }^{71}$ and replace saturated fats with unsaturated fats and experiment (Ethan Weiss, personal communication).

(FAODs) (online supplemental table $\mathrm{S} 1$ ). A special case of FAOD is CPT1A deficiency due to the so-called arctic variant, which is the most common CPT1A allele $(68 \%-$ $85 \%)$ in indigenous arctic populations but is extremely rare in the general population (box 1). In contrast to the mutations causing primary CPT1A deficiency, which completely abolish enzymatic function, the arctic variant has modulatory effects on CPT1A that decrease its baseline activity but increase its activation in the context of a diet high in omega-3 fatty acids and low in carbohydrates such as that traditionally consumed by Arctic populations. This might explain its positive selection in these populations and the potential risks that this variant may pose in view of the recent shift to a Western diet in the Arctic (box 2).

Other inborn conditions provide an indication for the use of $\mathrm{KD}$ as either a first-line therapy or adjunct therapy to improve symptoms and outcomes (online supplemental table S2). These conditions include glucose transporter 1 (GLUT1)-deficiency syndrome, pyruvate dehydrogenase (PDC) deficiency, drug-resistant epilepsy, glycogen storage disease, mitochondrial disorders, urea cycle disorders, purine metabolism disorders and amino acid metabolism disorders. Most of these conditions, with the exception of glycogen storage disease, present with seizures, impaired brain function and neurological symptoms that show significant improvements on KD through complex yet not fully elucidated mechanisms.

\section{TOWARDS EVIDENCE-BASED RECOMMENDATIONS}

There is still insufficient evidence for the use of genetic variants for the prediction of $\mathrm{KD}$ response. While some variants can have strong effects on $\mathrm{KD}$ response, these variants are found in rare congenital disorders and are therefore not relevant for the majority of the population (online supplemental tables S1 and S2). On the other hand, common SNPs that are widely represented in the population have small effects sizes and work together with other gene variants and lifestyle factors to affect complex trait such as KD response (table 1). Future studies with robust design and adequate power should replicate and quantify the associations observed in intervention studies of $\mathrm{KD}$ response, test additional promising candidate gene variants (online supplemental table S3), and identify new variants. This will ultimately facilitate the development of prediction models that quantify the contribution of each of these variants on KD outcomes as well as their interaction with dietary intake and other risk factors.

\section{Candidate genes}

Promising candidate SNPs include those associated with $\mathrm{KD}$ response in intervention trials (table 1), as well as additional SNPs presented in online supplemental table S3, which we have selected based on the two following criteria: (1) location within genes that are mutated in rare diseases with strong effects on the metabolism of ketones and fats (online supplemental tables S1 and S2) and (2) associations with metabolic traits in observational studies.

The discovery of new genetic variants in large welldesigned GWAS will be critical for the development of more accurate prediction models of $\mathrm{KD}$ response for personalised patient profiling. Data sets from large intervention GWAS could be used for predicting an individual's likelihood of successful response before the start of a $\mathrm{KD}$ intervention using models that integrate baseline factors (eg, age, height, sex, baseline weight) and outcome-related variables (eg, target outcomes such as macronutrient composition, total energy intake, expected weight loss, seizure reduction, etc). For example, a model of this kind was able to predict with a $\sim 80 \%$ accuracy the 


\section{Box 2 The 'Arctic variant of CPT1A}

\section{One of the strongest selective sweeps in human evolution}

The so-called 'arctic variant' of CPT1A (rs80356779 G>A; c.1436C >T or Pro479Leu on the reverse strand) is the most common allele $(68 \%$ to $85 \%$ ) in indigenous arctic populations (Alaska, Greenland, Northern Canada, and northeastern Siberia) ${ }^{72-76}$ but is absent in other publicly available genomes. ${ }^{72}$ A strong positive selection, a 'selective sweep', has driven this variant to high frequency in circum-Arctic populations, possibly as a result of the selective advantage it originally provided to a high-fat/low carbohydrate diet or cold environment. ${ }^{75}$

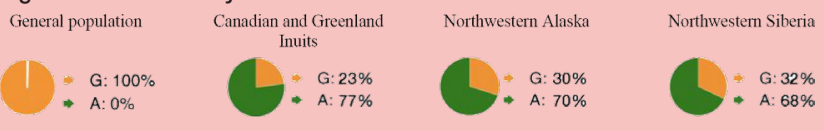

\section{Effects on enzyme function and dietary context}

In contrast to the mutations causing primary CPT1A deficiency, which completely abolish enzymatic function, the arctic variant has two counteracting effects on CPT1A activity that interact with dietary factors. While the variant induces a $25 \%-50 \%$ reduction in CPT1A activity, ${ }^{73}$ it also reduces CPT1A sensitivity to inhibition by malonyl-CoA in response to insulin signaling. ${ }^{77} 78$ As a result, given that the traditional arctic diet was rich in nutrients that activate CPT1A, the enzyme produced by the arctic variant might have been even more active than the wild-type one. ${ }^{78}$ The traditional arctic diet was not only high in fat and low in carbohydrates but also four times higher in omega-3 polyunsaturated fatty acids (PUFAs) compared with a standard Western diet. ${ }^{79}$ Omega-3 PUFAs are strong activators of CPT1A and have been shown to increase ketone levels twice as much as saturated fat in humans. ${ }^{80} 81$

\section{How ketogenic diet (KD) might have driven the selective sweep of the arctic variant}

What might have driven the selective sweep of the arctic variant? Beneficial health effects have been observed in Greenland Inuits (protection from atherosclerosis)and ${ }^{76}$ in Yup'ik Eskimos (lower adiposity with higher high-density lipoprotein) ${ }^{82}$ In contrast, homozygous infants eating non-traditional diets have higher mortality rate due to infectious disease and intolerance to fasting (lower ketone production and hypoglycaemia). ${ }^{83} 84$ This paradox might be explained by the need to adapt to long-term KD during evolution and the recent shift to a Western diet in the Arctic. One hypothesis, which frames ketosis as a potentially harmful state, is that the advantage of the arctic variant was to decrease ketosis through reduction of CPT1A activity thus preventing the risk of ketoacidosis. ${ }^{85}$ Another hypothesis is that the arctic variant would have enabled to maintain ketosis despite the high protein content of traditional Arctic diets ${ }^{86} 87$ through lower inhibition of CPT1A by malonyl$\mathrm{CoA}{ }^{73}$ If true, this second hypothesis would support the role of ketosis as essential biochemical state in the context of carbohydrate restriction. We still don't know which, if any, of these hypotheses may be true since most of the available evidence is based on studies in children eating non-traditional diets. In any case, it is likely that the traditional arctic diet might have provided an environment that maximised the beneficial effects of the arctic variant, whereas the shift to a Western diet might have unmasked its potential risks.

\section{Toward evidence-based recommendations for homozygous infants}

Rather than being a cause of infant death, the arctic variant is likely to be only one of a complex set of contributing factors due to the shift to a Western diet rich in refined carbohydrates and low in omega-3 PUFAs.

Continued

\section{Box 2 Continued}

A prospective cohort study is currently testing the hypothesis that preand postnatal exposure to n-3 PUFAs may reduce the adverse effects of the arctic variant in Alaska Native children. The results of this study may shed some light on the gene-diet interactions that might have driven the selective sweep of the arctic variant and facilitate the development of evidence-based recommendations for homozygous infants identified by newborn screening. ${ }^{88}$ Public recommendations to increase feeding with nontraditional carbohydrate-rich diets are not based on evidence and may have unintended harmful consequences. ${ }^{78}$

probability of losing $>5 \%$ of body weight after following for 1 year, one of four hypocaloric diets with carbohydrate composition ranging from $35 \%$ to $65 \%$ using data from the first 3 months of weight loss in the POUND-LOST trial. $^{33}$

\section{Polygenic scores}

The most relevant GWAS of KD response could also be used to develop polygenic scores, which enable greater power than individual SNPs for detection of interactions with environmental factors, including diet. Polygenic scores provide a more quantitative metric of an individual's genetic likelihood to express a given trait, such as a certain $\mathrm{KD}$ response, based on the cumulative impact of many common SNPs. Weights are generally assigned to each SNP according to the strength of its association with a given trait (effect estimate). Individuals are scored based on how many risk alleles they have for each variant (eg, zero, one, two copies) included in the polygenic score. Recent studies have shown that polygenic scores can greatly enhance the ability to identify clinically meaningful variations in the predisposition to common lifestyle-related diseases such as coronary artery disease, type 2 diabetes and obesity. ${ }^{34} 35$ These advances have been made possible thanks to the conduction of GWAS with large sample size enabling more precise effect estimates, the development of algorithms that combine genomewide sets of variants, and the availability of large biobanks for validation and testing. Using the same tools, it is possible to develop polygenic scores for the prediction of diet response that would complement those for disease risk prediction by facilitating the design of personalised diet interventions for individuals at higher polygenic risk for certain lifestyle-related diseases.

\section{Dynamic biomarkers}

Another promising approach is to apply a systems genetics framework to integrate polygenic gene prediction with dynamic biomarkers such as metabolic, epigenetic, transcriptomic profile and the microbiome to identify 'hub genes' that regulate gene networks interacting with environmental factors. These dynamic biomarkers rapidly respond to dietary manipulation and interact with both physiological factors (eg, sex, age) and lifestyle-related factors (IR, activity level, stress, etc). Once implemented, ketogenic therapies require oversight, especially in 
vulnerable patient populations. Therefore, biomarkers of compliance and efficacy that can be monitored by patients and their medical teams are important to develop. One such proposed tool is the Glucose:Ketone Index (GKI), a value used to describe the ratio of glucose to ketones (in $\mathrm{mmol} / \mathrm{L}$ ) in the blood of individuals on ketogenic metabolic therapies. Preliminary data from animals and small human trials suggest that GKI may successfully predict therapeutic efficacy of the KD in brain cancer. Similarly, subjective qualitative markers such as self-reported appetite, energy levels and sleep quality may also be used to predict candidacy, compliance and efficacy of ketogenic therapies. Impairments in one or more of such areas may support initiation, alteration, or discontinuance of the $\mathrm{KD}$, depending on the individual patient and their specific lifestyle and medical needs.

Since people vary widely in their response to a given amount of carbohydrate, biomarkers may be useful in determining who is most appropriate for a $\mathrm{KD}$, or what level of carbohydrate restriction is required to enable weight loss, disease reversal or other desired outcomes. One such marker is the monounsaturated fatty acid palmitoleic acid (cis-16:1n7). Palmitoleic acid is the primary fatty acid product of de novo lipogenesis (DNL). It is produced from palmitic acid by stearoyl CoA desaturase-1, increasingly proportionally more than any other fatty acid when carbohydrate is provided in excess driving up DNL. ${ }^{36}$ Since palmitoleic acid is relatively low in the diet, it's abundance can be useful as proxy of the metabolic pathway that converts carbohydrate to fat, with the caveat that very high intakes of specific palmitoleic acid-rich foods (eg, macadamia nuts and avocado) may confound this association. Beyond its role as a surrogate for DNL, greater abundance of palmitoleic acid in the circulation or in tissue membranes is strongly linked to a host of metabolic derangements including obesity, ${ }^{37}$ MetS, ${ }^{38}{ }^{39}$ type 2 diabetes, ${ }^{40-42}$ heart failure ${ }^{43} 44$ and CVD mortality. ${ }^{45} 46$ Thus, higher proportion of plasma palmitoleic acid is an early indication of carbohydrate intolerance (ie, more carbohydrate directed toward DNL), and an independent risk factor for diabetes and CVD.

We have reported in multiple studies that there is a remarkable stepwise uniformity in the response of circulating palmitoleic acid to varying level so of carbohydrate. Palmitoleic acid consistently decreases when carbohydrates are restricted, especially KDs, and increases when more carbohydrate is consumed. ${ }^{4-49}$ This effect is not significantly altered by weight loss or sex. Notable, is the fact that at any given level of carbohydrate intake palmitoleic acid levels vary, likely reflecting hereditary factors manifesting in an IR (ie, carbohydrate intolerant) phenotype. Whereas the direction of palmitoleic acid change is consistent as people add more carbohydrate to their diet, the magnitude varies. As such, if a person is able to maintain a relatively low level of palmitoleic acid it suggests they are 'appropriately' disposing of carbohydrate through oxidative pathways as opposed to DNL. In contrast, if palmitoleic acid levels are high or are rising it suggests that the current level of carbohydrate intake is not being managed in a healthy manner. In this way, palmitoleic acid could be used to titrate a personalised level of carbohydrate consumption.

\section{Establishing common standards of study design and variant interpretation}

While this review focuses on genetic variants of $\mathrm{KD}$ response, it is also a call to action for researchers, clinicians and funding institutions to establish common standards for study design and variant interpretation for the field of nutrigenomics as a whole. This will enable the building of a nutrigenomic knowledge base and accelerate the implementation of nutrigenomics in precision medicine.

The scientific validation of nutrigenetic variants will require the use of large sample size, common definitions for dietary protocols, and patient stratification to evaluate heterogeneity of response in different patient subgroups. Large sample sizes will minimise both false positive and false negative associations and enable the replication of the most significant associations across different studies. Reproducibility of results will also require the establishment of common standards to define what constitute a 'high-fat', 'low-carb' or 'ketogenic' diet—definitions that are highly variable from study to study-as the effects of high fat intake are heavily dependent on the quantity and quality of dietary carbohydrates. ${ }^{13}$ A valuable method of defining a $\mathrm{KD}$ may be one that elevates ketones above approximately $0.5 \mathrm{mM}$. Future studies of $\mathrm{KD}$ response should also evaluate modified KD protocols tailored to different subgroup of people with the goal of both personalising and expanding the applications of $\mathrm{KD}$ in clinical settings.

The clinical implementation of genetic variants of KD response, and nutrigenetic variants in general, will also require the development of a rigorous framework for variant interpretation. A model for such a framework is the partnership between ClinGen and ClinVar, two efforts of the National Institutes of Health to support genomic interpretation and implementation. The ClinGen programme employs Expert Panels to assess the clinical validity and actionability of disease-related gene variants, which are then shared with the public through the ClinVar database. Building a similar framework for the interpretation of nutrigenetics variants will require several steps including: (1) the development of common guidelines such as those proposed by Grimaldi $e t a l^{15}$ and establishment of Expert Panels for the identification of scientifically valid and clinically useful variants; (2) public sharing and crowdsourcing of variant submission from clinical testing labs, research institutions, public databases and professional societies; (3) the creation of working groups to refine and update guidelines as they are tested and deployed by the community. Taking these steps will greatly accelerate the implementation of nutrigenetics in clinical practice. 
Twitter Lucia Aronica @LuciaAronica and Dominic P D'agostino @DominicDAgosti2

Acknowledgements We thank Annalouise 0' Connor at Metagenics Inc. for providing critical input on the analysis plan and data report.

Contributors LA conceived and designed the analysis, collected the data and wrote the manuscript draft. JV, AP and DPD provided key data and content and critically reviewed the manuscript.

Funding DPD is supported by the Office of Naval Research (ONR), U.S. Department of Defense [grant number: N000141310062]

Competing interests JV is a founder and equity holder in Virta Health, scientific advisors for Atkins Nutritionals and UCAN, and receives royalties from books on ketogenic diets. AP is coowner of the company Poff Medical Consulting and Communications which provides scientific consulting services. AP is also coowner of the company Metabolic Health Initiative, LLC which hosts the annual scientific conference Metabolic Health Summit. AP is an inventor on patents related to ketone technology. DPD is an inventor on International Patent \# PCT/US2014/031237 University of South Florida: Compositions and Methods for Producing Elevated and Sustained Ketosis. DPD is a coowner of the company Ketone Technologies, a company specialised in scientific consulting.

Patient consent for publication Not required.

Provenance and peer review Not commissioned; externally peer reviewed by Melina B Jampolis MD, Heali AI, USA and Wesley Kephart, University of WisconsinWhitewater, USA.

Data availability statement All data relevant to the study are included in the article or uploaded as online supplemental information.

Supplemental material This content has been supplied by the author(s). It has not been vetted by BMJ Publishing Group Limited (BMJ) and may not have been peer-reviewed. Any opinions or recommendations discussed are solely those of the author(s) and are not endorsed by BMJ. BMJ disclaims all liability and responsibility arising from any reliance placed on the content. Where the content includes any translated material, BMJ does not warrant the accuracy and reliability of the translations (including but not limited to local regulations, clinical guidelines, terminology, drug names and drug dosages), and is not responsible for any error and/or omissions arising from translation and adaptation or otherwise.

Open access This is an open access article distributed in accordance with the Creative Commons Attribution Non Commercial (CC BY-NC 4.0) license, which permits others to distribute, remix, adapt, build upon this work non-commercially, and license their derivative works on different terms, provided the original work is properly cited, appropriate credit is given, any changes made indicated, and the use is non-commercial. See: http://creativecommons.org/licenses/by-nc/4.0/.

\section{ORCID iD}

Lucia Aronica http://orcid.org/0000-0002-8991-4858

\section{REFERENCES}

1 Loos RJF. The genetics of adiposity. Curr Opin Genet Dev 2018:50:86-95

2 Ghosh S, Bouchard C. Convergence between biological, behavioura and genetic determinants of obesity. Nat Rev Genet 2017;18:731-48

3 Rauschert S, Kirchberg FF, Marchioro L, et al. Early programming of obesity throughout the life course: a metabolomics perspective. Ann Nutr Metab 2017;70:201-9.

4 Heianza Y, Qi L. Impact of genes and environment on obesity and cardiovascular disease. Endocrinology 2019;160:81-100.

5 PETERMAN MG. The ketogenic diet. JAMA 1928;90:1427-9.

6 Kossoff EH, Zupec-Kania BA, Auvin S, et al. Optimal clinical management of children receiving dietary therapies for epilepsy: updated recommendations of the International ketogenic diet Study Group. Epilepsia Open 2018;3:175-92.

7 Sato K, Kashiwaya Y, Keon CA, et al. Insulin, ketone bodies, and mitochondrial energy transduction. The FASEB Journal 1995;9:651-8.

8 Ludwig DS, Willett WC, Volek JS, et al. Dietary fat: from foe to Friend? Science 2018;362:764-70.

9 Saslow LR, Daubenmier JJ, Moskowitz JT, et al. Twelve-month outcomes of a randomized trial of a moderate-carbohydrate versus very low-carbohydrate diet in overweight adults with type 2 diabetes mellitus or prediabetes. Nutr Diabetes 2017;7:304

10 Hallberg SJ, McKenzie AL, Williams PT, et al. Author correction: effectiveness and safety of a novel care model for the management of type 2 diabetes at 1 year: an open-label, Non-Randomized, controlled study. Diabetes Therapy 2018;9:613-21.

11 Athinarayanan SJ, Adams RN, Hallberg SJ, et al. Long-Term effects of a novel continuous remote care intervention including nutritional ketosis for the management of type 2 diabetes: a 2-year Nonrandomized clinical trial. Front Endocrinol 2019;10:348.

12 Westman EC, Feinman RD, Mavropoulos JC, et al. LowCarbohydrate nutrition and metabolism. Am J Clin Nutr 2007;86:276-84.

13 Zilberter T, Zilberter Y. Ketogenic ratio determines metabolic effects of macronutrients and prevents interpretive bias. Front Nutr 2018;5:75.

14 Tai ES, Corella D, Demissie S, et al. Polyunsaturated fatty acids interact with the PPARA-L162V polymorphism to affect plasma triglyceride and apolipoprotein C-III concentrations in the Framingham heart study. J Nutr 2005;135:397-403.

15 Grimaldi KA, van Ommen B, Ordovas JM, et al. Proposed guidelines to evaluate scientific validity and evidence for genotype-based dietary advice. Genes Nutr 2017;12:35.

16 Ruaño G, Windemuth A, Kocherla M, et al. Physiogenomic analysis of weight loss induced by dietary carbohydrate restriction. Nutr Metab 2006;3:20.

17 Seip RL, Volek JS, Windemuth A, et al. Physiogenomic comparison of human fat loss in response to diets restrictive of carbohydrate or fat. Nutr Metab 2008:5:4.

18 Yvan-Charvet L, Even P, Bloch-Faure M, et al. Deletion of the angiotensin type 2 receptor (AT2R) reduces adipose cell size and protects from diet-induced obesity and insulin resistance. Diabetes 2005;54:991-9.

19 Yang Q, Graham TE, Mody N, et al. Serum retinol binding protein 4 contributes to insulin resistance in obesity and type 2 diabetes. Nature 2005;436:356-62.

20 Lecklin A, Tuomisto L, MacDonald E. Metoprine, an inhibitor of histamine N-methyltransferase but not catechol-O-methyltransferase suppresses feeding in sated and in food deprived rats. Methods Find Exp Clin Pharmacol 1995;17:47-52.

21 Schoeler NE, Leu C, Balestrini S, et al. Genome-Wide association study: exploring the genetic basis for responsiveness to ketogenic dietary therapies for drug-resistant epilepsy. Epilepsia 2018;59:1557-66.

22 Liu C-C, Liu C-C, Kanekiyo T, et al. Apolipoprotein E and Alzheimer disease: risk, mechanisms and therapy. Nat Rev Neurol 2013;9:106-18.

23 Gureje O, Ogunniyi A, Baiyewu O, et al. APOE $\varepsilon 4$ is not associated with Alzheimer's disease in elderly Nigerians. Ann Neurol 2006;59:182-5

$24 \mathrm{Bu}$ G. Apolipoprotein E and its receptors in Alzheimer's disease: pathways, pathogenesis and therapy. Nat Rev Neurosci 2009;10:333-44.

25 Craft S, Asthana S, Schellenberg G, et al. Insulin effects on glucose metabolism, memory, and plasma amyloid precursor protein in Alzheimer's disease differ according to apolipoprotein-E genotype. Ann N Y Acad Sci 2000;903:222-8.

26 Viola KL, Klein WL. Amyloid $\beta$ oligomers in Alzheimer's disease pathogenesis, treatment, and diagnosis. Acta Neuropathol 2015;129:183-206.

27 Wu L, Zhang X, Zhao L. Human APOE isoforms differentially modulate brain glucose and ketone body metabolism: implications for Alzheimer's disease risk reduction and early intervention. $J$ Neurosci 2018;38:6665-81.

28 Nagpal R, Neth BJ, Wang S, et al. Modified Mediterranean-ketogenic diet modulates gut microbiome and short-chain fatty acids in association with Alzheimer's disease markers in subjects with mild cognitive impairment. EBioMedicine 2019;47:529-42.

29 Morrill SJ, Gibas KJ. Ketogenic diet rescues cognition in ApoE4+ patient with mild Alzheimer's disease: a case study. Diabetes Metab Syndr 2019;13:1187-91.

30 Gardener SL, Rainey-Smith SR, Sohrabi HR, et al. Increased carbohydrate intake is associated with poorer performance in verbal memory and attention in an APOE genotype-dependent manner. $J$ Alzheimers Dis 2017;58:193-201.

31 Reger MA, Henderson ST, Hale C, et al. Effects of $\beta$-hydroxybutyrate on cognition in memory-impaired adults. Neurobiol Aging 2004:25:311-4.

32 Henderson ST, Vogel JL, Barr LJ, et al. Study of the ketogenic agent AC-1202 in mild to moderate Alzheimer's disease: a randomized, double-blind, placebo-controlled, multicenter trial. Nutr Metab 2009;6:31.

33 Thomas DM, Ivanescu AE, Martin CK, et al. Predicting successful long-term weight loss from short-term weight-loss outcomes: new insights from a dynamic energy balance model (the pounds lost study). Am J Clin Nutr 2015;101:449-54. 
34 Khera AV, Chaffin M, Aragam KG, et al. Genome-Wide polygenic scores for common diseases identify individuals with risk equivalent to monogenic mutations. Nat Genet 2018;50:1219-24.

35 Khera AV, Chaffin M, Wade $\mathrm{KH}$, et al. Polygenic prediction of weight and obesity trajectories from birth to adulthood. Cell 2019;177:e9:587-96.

36 Aarsland A, Wolfe RR. Hepatic secretion of VLDL fatty acids during stimulated lipogenesis in men. J Lipid Res 1998;39:1280-6.

37 Smith CE, Tucker KL, Arnett DK, et al. Apolipoprotein A2 polymorphism interacts with intakes of dairy foods to influence body weight in 2 U.S. populations. J Nutr 2013;143:1865-71.

38 Paillard F, Catheline D, Duff FL, et al. Plasma palmitoleic acid, a product of stearoyl-coA desaturase activity, is an independent marker of triglyceridemia and abdominal adiposity. Nutr Metab Cardiovasc Dis 2008;18:436-40.

39 Warensjö E, Risérus U, Vessby B. Fatty acid composition of serum lipids predicts the development of the metabolic syndrome in men. Diabetologia 2005;48:1999-2005.

40 Wang L, Folsom AR, Zheng Z-J, et al. Plasma fatty acid composition and incidence of diabetes in middle-aged adults: the Atherosclerosis risk in communities (ARIC) study. Am J Clin Nutr 2003;78:91-8.

41 Mahendran Y, Ågren J, Uusitupa M, et al. Association of erythrocyte membrane fatty acids with changes in glycemia and risk of type 2 diabetes. Am J Clin Nutr 2014;99:79-85.

42 Vessby B, Aro A, Skarfors E, et al. The risk to develop NIDDM is related to the fatty acid composition of the serum cholesterol esters. Diabetes 1994;43:1353-7.

43 Djoussé L, Weir NL, Hanson NQ, et al. Plasma Phospholipid Concentration of Cis -Palmitoleic Acid and Risk of Heart Failure. Circulation 2012;5:703-9.

44 Yamagishi K, Nettleton JA, Folsom AR. Plasma fatty acid composition and incident heart failure in middle-aged adults: the Atherosclerosis risk in communities (ARIC) study. Am Heart $J$ 2008;156:965-74.

45 Warensjö E, Sundström J, Vessby B, et al. Markers of dietary fat quality and fatty acid desaturation as predictors of total and cardiovascular mortality: a population-based prospective study. Am J Clin Nutr 2008;88:203-9.

46 Djoussé L, Matthan NR, Lichtenstein AH, et al. Red blood cell membrane concentration of cis-palmitoleic and cis-vaccenic acids and risk of coronary heart disease. Am J Cardiol 2012;110:539-44.

47 Volek JS, Phinney SD, Forsythe CE, et al. Carbohydrate restriction has a more favorable impact on the metabolic syndrome than a low fat diet. Lipids 2009;44:297-309.

48 Volk BM, Kunces LJ, Freidenreich DJ, et al. Effects of step-wise increases in dietary carbohydrate on circulating saturated fatty acids and palmitoleic acid in adults with metabolic syndrome. PLoS One 2014;9:e113605.

49 Hyde PN, Sapper TN, Crabtree CD, et al. Dietary carbohydrate restriction improves metabolic syndrome independent of weight loss. JCl Insight 2019;4. doi:10.1172/jci.insight.128308. [Epub ahead of print: 2006 2019].

50 Smith CE, Van Rompay MI, Mattei J, et al. Dietary fat modulation of hepatic lipase variant $-514 \mathrm{C} / \mathrm{T}$ for lipids: a crossover randomized dietary intervention trial in Caribbean Hispanics. Physiol Genomics 2017;49:592-600.

51 Tai ES, Corella D, Deurenberg-Yap M, et al. Dietary Fat Interacts with the $-514 \mathrm{C}>\mathrm{T}$ Polymorphism in the Hepatic Lipase Gene Promoter on Plasma Lipid Profiles in a Multiethnic Asian Population: The 1998 Singapore National Health Survey. J Nutr 2003;133:3399-408

52 Ordovas JM, Corella D, Demissie S, et al. Dietary fat intake determines the effect of a common polymorphism in the hepatic lipase gene promoter on high-density lipoprotein metabolism: evidence of a strong dose effect in this gene-nutrient interaction in the Framingham study. Circulation 2002;106:2315-21.

53 Corella D, Arnett DK, Tsai MY, et al. The $-256 \mathrm{~T}>\mathrm{C}$ polymorphism in the apolipoprotein A-II gene promoter is associated with body mass index and food intake in the genetics of lipid lowering drugs and diet network study. Clin Chem 2007:53:1144-52.

54 Corella D, Peloso G, Arnett DK, et al. APOA2, dietary fat, and body mass index: replication of a gene-diet interaction in 3 independent populations. Arch Intern Med 2009;169:1897-906.

55 Delgado-Lista J, Perez-Jimenez F, Tanaka T, et al. An apolipoprotein A-II polymorphism (-265T/C, rs5082) regulates postprandial response to a saturated fat overload in healthy men. J Nutr 2007;137:2024-8.

56 Jansen S, Lopez-Miranda J, Salas J, et al. Effect of 347-serine mutation in apoprotein A-IV on plasma LDL cholesterol response to dietary fat. Arterioscler Thromb Vasc Biol 1997;17:1532-8.

57 Bouchard-Mercier A, Godin G, Lamarche B, et al. Effects of peroxisome proliferator-activated receptors, dietary fat intakes and gene-diet interactions on peak particle diameters of low-density lipoproteins. J Nutrigenet Nutrigenomics 2011;4:36-48.

58 Tai ES, Demissie S, Cupples LA, et al. Association between the ppara L162V polymorphism and plasma lipid levels. Arterioscler Thromb Vasc Biol 2002;22:805-10.

59 Musunuru K, Strong A, Frank-Kamenetsky M, et al. From noncoding variant to phenotype via SORT1 at the 1p13 cholesterol locus. Nature 2010;466:714-9.

60 Talmud PJ, Shah S, Whittall R, et al. Use of low-density lipoprotein cholesterol gene score to distinguish patients with polygenic and monogenic familial hypercholesterolaemia: a case-control study. Lancet 2013;381:1293-301.

61 Wang J, Dron JS, Ban MR, et al. Polygenic versus monogenic causes of hypercholesterolemia ascertained clinically. Arterioscler Thromb Vasc Biol 2016;36:2439-45.

62 Teslovich TM, Musunuru K, Smith AV, et al. Biological, clinical and population relevance of 95 loci for blood lipids. Nature 2010;466:707-13.

63 Chora JR, Alves AC, Medeiros AM, et al. Lysosomal acid lipase deficiency: a hidden disease among cohorts of familial hypercholesterolemia? J Clin Lipidol 2017;11:477-84.

64 Berge KE, Tian H, Graf GA, et al. Accumulation of dietary cholesterol in sitosterolemia caused by mutations in adjacent $A B C$ transporters. Science 2000;290:1771-5.

65 Sturm AC, Knowles JW, Gidding SS, et al. Clinical Genetic Testing for Familial Hypercholesterolemia: JACC Scientific Expert Panel. J Am Coll Cardiol 2018;72:662-80.

66 Chora JR, Medeiros AM, Alves AC, et al. Analysis of publicly available LDLR, ApoB, and PCSK9 variants associated with familial hypercholesterolemia: application of ACMG guidelines and implications for familial hypercholesterolemia diagnosis. Genet Med 2018;20:591-8.

67 Bhanpuri NH, Hallberg SJ, Williams PT, et al. Cardiovascular disease risk factor responses to a type 2 diabetes care model including nutritional ketosis induced by sustained carbohydrate restriction at 1 year: an open label, non-randomized, controlled study. Cardiovasc Diabetol 2018;17:56

68 Sharman MJ, Kraemer WJ, Love DM, et al. A ketogenic diet favorably affects serum biomarkers for cardiovascular disease in normalweight men. J Nutr 2002;132:1879-85

69 Sharman MJ, Gómez AL, Kraemer WJ, et al. Very low-carbohydrate and low-fat diets affect fasting lipids and postprandial lipemia differently in overweight men. J Nutr 2004;134:880-5.

70 Krauss RM, Blanche PJ, Rawlings RS, et al. Separate effects of reduced carbohydrate intake and weight loss on atherogenic dyslipidemia. Am J Clin Nutr 2006;83:1025-31.

71 Mason AE, Saslow LR, Moran PJ, et al. Lipid findings from the diabetes education to lower insulin, sugars, and hunger (DELISH) study. Nutr Metab 2019;16:58.

72 , Abecasis GR, Auton A, et al, 1000 Genomes Project Consortium. An integrated map of genetic variation from 1,092 human genomes. Nature 2012;491:56-65.

73 Greenberg CR, Dilling LA, Thompson GR, et al. The paradox of the carnitine palmitoyltransferase type la P479L variant in Canadian Aboriginal populations. Mol Genet Metab 2009;96:201-7.

74 Gessner BD, Gillingham MB, Johnson MA, et al. Prevalence and distribution of the $\mathrm{C} .1436 \mathrm{C} \rightarrow \mathrm{T}$ sequence variant of carnitine palmitoyltransferase $1 \mathrm{~A}$ among Alaska native infants. J Pediatr 2011;158:124-9.

75 Clemente FJ, Cardona A, Inchley CE, et al. A selective sweep on a deleterious mutation in CPT1a in Arctic populations. Am J Hum Genet 2014;95:584-9.

76 Rajakumar C, Ban MR, Cao H, et al. Carnitine palmitoyltransferase la polymorphism P479L is common in Greenland Inuit and is associated with elevated plasma apolipoprotein A-I. J Lipid Res 2009;50:1223-8

77 Brown NF, Mullur RS, Subramanian I, et al. Molecular characterization of L-CPT I deficiency in six patients: insights into function of the native enzyme. J Lipid Res 2001;42:1134-42.

78 Fohner AE, Garrison Nanibaa' A, Austin MA, et al. Carnitine palmitoyltransferase $1 \mathrm{~A}$ P479L and infant death: policy implications of emerging data. Genet Med 2017;19:851-7

79 Bang HO, Dyerberg J, Sinclair HM. The composition of the Eskimo food in North Western Greenland. Am J Clin Nutr 1980;33:2657-61.

80 Fuehrlein BS, Rutenberg MS, Silver JN, et al. Differential Metabolic Effects of Saturated Versus Polyunsaturated Fats in Ketogenic Diets. J Clin Endocrinol Metab 2004;89:1641-5.

81 Cunnane SC. Metabolism of polyunsaturated fatty acids and ketogenesis: an emerging connection. Prostaglandins Leukot Essent Fatty Acids 2004;70:237-41. 
82 Lemas DJ, Wiener HW, O'Brien DM, et al. Genetic polymorphisms in carnitine palmitoyltransferase $1 \mathrm{~A}$ gene are associated with variation in body composition and fasting lipid traits in Yup'ik Eskimos. J Lipid Res 2012;53:175-84.

83 Gillingham MB, Hirschfeld M, Lowe S, et al. Impaired fasting tolerance among Alaska native children with a common carnitine palmitoyltransferase 1A sequence variant. Mol Genet Metab 2011;104:261-4.

84 Gessner BD, Gillingham MB, Birch S, et al. Evidence for an association between infant mortality and a carnitine palmitoyltransferase 1A genetic variant. Pediatrics 2010;126:945-51.
85 Joshi S, Ostfeld RJ, McMacken M. The ketogenic diet for obesity and Diabetes - Enthusiasm Outpaces evidence. JAMA Intern Med 2019;179:1163.

86 Sinclair HM. The diet of Canadian Indians and Eskimos. Proc Nutr Soc 1953;12:69-82.

87 Heinbecker P. Further studies on the metabolism of Eskimos. J Biol Chem 1931;93:327-36.

88 Koeller DMD, Denise A. Diet and the CPT1A arctic variant: Impact on the Health of Alaska Native Children Grantome - NIH. Available: https://grantome.com/grant/NIH/R01-HD089951-03 